\title{
KILLING SYMMETRY IN SPECIAL AXIALLY SYMMETRIC STATIC SPACETIMES IN TELEPARALEL THEORY OF GRAVITATION
}

\author{
${ }^{1}$ AMJAD ALI AND ${ }^{2}$ TARIQ ZAMAN KHAN \\ ${ }^{1,2}$ Department of Basic Sciences and Islamiat, University of Engineering \& \\ Technology Peshawar, Pakistan. \\ Email: ${ }^{1}$ amjad_puet@yahoo.com, ${ }^{2}$ tariqnsdc@gmail.com
}

\begin{abstract}
Revised November 2015
ABSTRACT. In this paper we are searching for teleparallel Killing vector fields of special axially symmetric static spacetimes in teleparallel theory of gravitation by using direct integration and algebraic techniques. After thorough investigations, the whole problem is divided into three cases under different constraints. Two of the said cases give contradiction, while one of them gives the solution of the system comprising the killing equations in the form of killing vector fields. The dimension of the killing symmetry in this case is 10.

Keywords: Lie derivative in teleparallel theory, covariant tensor, teleparallel killing symmetry.
\end{abstract}

1. Introduction. The importance of symmetry in both the theory of general relativity and teleparallel theory of gravitation is pretty clear as many physical laws in the universe are direct applications of symmetry in all types of relativity theories, that is in special, general and teleparallel theory of gravitation. Many researchers made their contributions in different types of symmetry and found a lot of hidden information of the universe by using different models of symmetry. Actually Einstein field equations give a relation between geometry and physics of the universe and symmetries play a vital role in the classification of solutions of Einstein field equations. Symmetry limitations are extremely supportive. During the last three decades, a lot of concentration has been given to the knowledge of different types of symmetries. One feature that helps us to recognize and expose the unseen realities of the space is the knowledge of conservation laws of a spacetime for the metric. As the conservation laws are attainable from the symmetry of spacetime [1], Killing, homothetic, conformal Killing and self similar symmetries of a spacetimes are extensively studied in the existence of curvature [2-11]. It has been noticed in the earlier period that for the spacetime having torsion only, the law of gravitation can be equally described [12]. M. Sharif et. al [13] introduced the concept of Killing symmetry in teleparallel theory and they got Killing vector fields for the Einstein space. In general relativity Killing vector fields are considered as an essential symmetry. In the background of teleparallel theory, a lot of work has been finished in obtaining Killing vector fields for different spacetimes [14-22]. To understand the physical and geometrical feature of our unexplained universe, much work is required to be done through symmetries in the presence of curvature or torsion only.

2. Overview of teleparallel theory. For a covariant tensor of rank 2 in teleparallel theory, the covariant derivative $\nabla_{\rho}$ is defined as [23]:

$$
\nabla_{\rho} C_{\mu v}=C_{\mu v, \rho}-\Gamma_{\rho \mu}^{\theta} C_{\mu \theta}-\Gamma_{\mu \rho}^{\theta} C_{v \theta},
$$

where comma is used for partial derivative and Weitzenböck connection $\Gamma_{\mu \rho}^{\theta}$ is defined by [23]:

$$
\Gamma_{\mu \nu}^{\theta}=S_{a}^{\theta} \partial_{\nu} S_{\mu}^{a}
$$

The non trivial tetrad field $S_{\mu}^{a}$ and its inverse field $S_{a}^{v}$ satisfy the relations: 
which generates metric tensor as

$$
S_{\mu}^{a} S_{a}^{v}=\delta_{\mu}^{v}, \quad S_{\mu}^{a} S_{b}^{v}=\delta_{b}^{a},
$$

$$
g_{\mu v}=\eta_{a b} S_{\mu}^{a} S_{v}^{b}
$$

$\eta_{a b}=\operatorname{diag}(-1,1,1,1)$ and $\eta_{a b}$ is the Minkowski metric. The torsion tensor defined as [23]

$$
T_{\mu v}^{\theta}=\Gamma_{\nu \mu}^{\theta}-\Gamma_{\mu v}^{\theta}
$$

Now the vector field $\mathrm{X}$ is said to be teleparallel killing vector field if it satisfies the equation

$$
L_{x}^{T} g_{\mu \nu}=g_{\mu v, \rho} X^{\rho}+g_{\rho v} X_{, \mu}^{\rho}+g_{\rho \mu} X_{, \nu}^{\rho}+X^{\rho}\left(g_{v \theta} T_{\mu \rho}^{\theta}+g_{\mu \theta} T_{v \rho}^{\theta}\right)=0,
$$

where $L_{X}^{T}$ denotes the Lie derivative in teleparallel theory of gravitation along the vector field X.

3. Main Result. Consider the special axially symmetric spacetime in usual coordinates $(t, r, \theta, \phi)$, labeled respectively by $\left(x^{0}, x^{1}, x^{2}, x^{3}\right)$, having the line element [24]:

$$
d s^{2}=-e^{A(r, \theta)} d t^{2}+e^{B(r, \theta)}\left(d r^{2}+d \theta^{2}+d \phi^{2}\right)
$$

Here $A(r, \theta)$ and $B(r, \theta)$ are function of $r$ and $\theta$ only. The two linearly independent Killing vector fields in general relativity admitted by the spacetime (7) are $\frac{\partial}{\partial t}$ and $\frac{\partial}{\partial \phi}$. The components of tetrad and its inverse can be found by using equation (3) as follows

$$
\begin{aligned}
S_{\mu}^{a} & =\left(\begin{array}{cccc}
e^{\frac{1}{2} A(r, \theta)} & 0 & 0 & 0 \\
0 & e^{\frac{1}{2} B(r, \theta)} & 0 & 0 \\
0 & 0 & e^{\frac{1}{2} B(r, \theta)} & 0 \\
0 & 0 & 0 & e^{\frac{1}{2} B(r, \theta)}
\end{array}\right), \\
S_{a}^{\mu} & =\left(\begin{array}{cccc}
e^{-\frac{1}{2} A(r, \theta)} & 0 & 0 & 0 \\
0 & e^{-\frac{1}{2} B(r, \theta)} & 0 & 0 \\
0 & 0 & e^{-\frac{1}{2} B(r, \theta)} & 0 \\
0 & 0 & 0 & e^{-\frac{1}{2} B(r, \theta)}
\end{array}\right)
\end{aligned}
$$

It can be confirmed simply that equation (3) and (4) are satisfied by $S_{\mu}^{a}$ and $S_{a}^{\mu}$. Now by using relation (2), the non vanishing Weitzenböck connection are obtained as:

$$
\begin{aligned}
& \Gamma_{01}^{0}=\frac{1}{2} A_{r}(r, \theta), \Gamma_{02}^{0}=\frac{1}{2} A_{\theta}(r, \theta), \Gamma_{12}^{1}=\frac{1}{2} B_{\theta}(r, \theta), \\
& \Gamma_{21}^{2}=\frac{1}{2} B_{r}(r, \theta), \Gamma_{31}^{3}=\frac{1}{2} B_{r}(r, \theta), \quad \Gamma_{32}^{3}=\frac{1}{2} B_{\theta}(r, \theta) .
\end{aligned}
$$

So the non vanishing torsion components can be obtained by using equation (5), which are: 


$$
\begin{aligned}
& T_{10}^{0}=-T_{01}^{0}=\frac{1}{2} A_{r}(r, \theta), \quad T_{20}^{0}=-T_{02}^{0}=\frac{1}{2} A_{\theta}(r, \theta), \quad T_{21}^{1}=-T_{12}^{1}=\frac{1}{2} B_{\theta}(r, \theta), \\
& T_{12}^{2}=-T_{21}^{2}=\frac{1}{2} B_{r}(r, \theta), \quad T_{13}^{3}=-T_{31}^{3}=\frac{1}{2} B_{r}(r, \theta), \quad T_{23}^{3}=-T_{32}^{3}=\frac{1}{2} B_{\theta}(r, \theta) .
\end{aligned}
$$

Now using equation (7) and (10) in equation (6), we get the following equations

$$
\begin{aligned}
& X_{, 0}^{0}=0 \\
& 2 e^{B(r, \theta)} X_{, 0}^{1}-2 e^{A(r, \theta)} X_{, 1}^{0}-A_{r}(r, \theta) e^{A(r, \theta)} X^{0}=0 \\
& 2 e^{B(r, \theta)} X_{, 0}^{2}-2 e^{A(r, \theta)} X_{, 2}^{0}-A_{\theta}(r, \theta) e^{A(r, \theta)} X^{0}=0 \\
& e^{B(r, \theta)} X_{, 0}^{3}-e^{A(r, \theta)} X_{, 3}^{0}=0 \\
& B_{r}(r, \theta) X^{1}+2 X_{, 1}^{1}=0 \\
& 2 X_{, 1}^{2}+2 X_{, 2}^{1}+B_{r}(r, \theta) X^{2}+B_{\theta}(r, \theta) X^{1}=0 \\
& \quad 2 X_{, 1}^{3}+2 X_{, 3}^{1}+B_{r}(r, \theta) X^{3}=0 \\
& B_{\theta}(r, \theta) X^{2}+2 X_{, 2}^{2}=0 \\
& \quad 2 X_{, 2}^{3}+2 X_{, 3}^{2}+B_{\theta}(r, \theta) X^{3}=0 \\
& X_{, 3}^{3}=0 .
\end{aligned}
$$

To find the Killing vector fields in teleparallel theory of gravitation, we proceed as follows: The following system is formed when we solve equations (11), (12), (19) and (20).

$$
\begin{aligned}
& X^{0}=F^{1}(r, \theta, \phi), \\
& X^{1}=t e^{A(r, \theta)-B(r, \theta)} F_{r}^{1}(r, \theta, \phi)+\frac{t}{2} A_{r}(r, \theta) e^{A(r, \theta)-B(r, \theta)} F^{1}(r, \theta, \phi)+F^{3}(r, \theta, \phi), \\
& X^{2}=-\phi F_{\theta}^{2}(t, r, \theta)-\frac{\phi}{2} B_{\theta}(r, \theta) F^{2}(t, r, \theta)+F^{4}(t, r, \theta), \\
& X^{3}=F^{2}(t, r, \theta) .
\end{aligned}
$$

where $F^{1}(r, \theta, \phi), F^{2}(t, r, \theta), F^{3}(r, \theta, \phi)$ and $F^{4}(t, r, \theta)$ are the functions of integration. By using rest of the equations, we can find the values of these functions so that the above system becomes 


$$
\begin{aligned}
X^{0}= & \phi c_{2} e^{\frac{1}{4} B(r, \theta)-\frac{3}{4} A(r, \theta)}+K^{2}(r, \theta), \\
X^{1}= & \frac{\phi t c_{2}}{4} e^{\frac{1}{4} A(r, \theta)-\frac{3}{4} B(r, \theta)}\left\{B_{r}(r, \theta)-A_{r}(r, \theta)\right\}+t e^{A(r, \theta)-B(r, \theta)} K_{r}^{2}(r, \theta)+\frac{t}{2} A_{r}(r, \theta) e^{A(r, \theta)-B(r, \theta)} K^{2}(r, \theta)+ \\
& \phi c_{4} e^{-\frac{1}{2} B(r, \theta)}+\left(\theta c_{7}+c_{8}\right) e^{-\frac{1}{2} B(r, \theta)} \\
X^{2}= & \frac{\phi t c_{2}}{4} e^{\frac{1}{4} A(r, \theta)-\frac{3}{4} B(r, \theta)}\left\{B_{\theta}(r, \theta)-A_{\theta}(r, \theta)\right\}-\phi c_{9} e^{-\frac{1}{2} B(r, \theta)}+t e^{-\frac{1}{2} B(r, \theta)+L^{4}(r)}+\left(r c_{5}+c_{6}\right) e^{-\frac{1}{2} B(r, \theta)} \\
X^{3}= & t c_{2} e^{\frac{1}{4} A(r, \theta)-\frac{3}{4} B(r, \theta)}+\left(\theta c_{9}+c_{10}\right) e^{-\frac{1}{2} B(r, \theta)}-r c_{4} e^{-\frac{1}{2} B(r, \theta)} .
\end{aligned}
$$

Using equation (22) in equation (15), we have

$$
\left\{B_{r}(r, \theta)-A_{r}(r, \theta)\right\}^{2}=4\left\{B_{r r}(r, \theta)-A_{r r}(r, \theta)\right\} .
$$

Similarly by using equation (22) in equation (18), we obtain

$$
\left\{B_{\theta}(r, \theta)-A_{\theta}(r, \theta)\right\}^{2}=4\left\{B_{\theta \theta}(r, \theta)-A_{\theta \theta}(r, \theta)\right\} .
$$

Suppose $\quad B_{r}(r, \theta)-A_{r}(r, \theta) \neq 0, \quad$ solving equation $\quad$ (23) we have $A(r, \theta)-B(r, \theta)=4 \log \left(r+L^{7}(\theta)\right)+L^{8}(\theta)$, using system of equations (22) and solving equation (8) and then equation (6) after a lengthy calculation leads to contradiction that $e^{c_{1}+c_{12}}=0$, so consider $\quad B_{\theta}(r, \theta)-A_{\theta}(r, \theta) \neq 0 \quad$ then implies $A(r, \theta)-B(r, \theta)=4 \log \left(\theta+L^{9}(r)\right)+L^{10}(r)$, again using system of equations (22) and solving equation (5) and then equation (6), which again leads to contradiction that $e^{c_{1}+c_{18}}=0$, Now taking $B_{r}(r, \theta)-A_{r}(r, \theta)=0$ and $B_{\theta}(r, \theta)-A_{\theta}(r, \theta)=0$, using equation (23) and equation (24), we have $A(r, \theta)=B(r, \theta)+c_{23}$, then after solving the remaining equations and by using system (22) we get the following system:

$$
\begin{aligned}
& X^{0}=\left(\phi c_{28}+\theta c_{25}+r c_{26}+c_{27}\right) e^{\frac{1}{2} B(r, \theta)-A(r, \theta)}, \quad X^{1}=\left(t c_{26}+\phi c_{4}-\theta c_{5}+c_{8}\right) e^{-\frac{1}{2} B(r, \theta)}, \\
& X^{2}=\left(t c_{25}-\phi c_{9}+r c_{5}+c_{6}\right) e^{-\frac{1}{2} B(r, \theta)}, \quad X^{3}=\left(t c_{28}-r c_{4}+\theta c_{9}+c_{10}\right) e^{-\frac{1}{2} B(r, \theta)} .
\end{aligned}
$$

The generators of the Killing vector fields mentioned in the above solution (25) are as;

$$
\begin{aligned}
& X_{1}=e^{-\frac{1}{2} B(r, \theta)}\left(\phi e^{B(r, \theta)-A(r, \theta)} \frac{\partial}{\partial t}+t \frac{\partial}{\partial \phi}\right), \quad X_{2}=e^{-\frac{1}{2} B(r, \theta)}\left(\theta e^{B(r, \theta)-A(r, \theta)} \frac{\partial}{\partial t}+t \frac{\partial}{\partial \theta}\right) \\
& X_{3}=e^{-\frac{1}{2} B(r, \theta)} \frac{\partial}{\partial r}, \quad X_{4}=e^{\frac{1}{2} B(r, \theta)-A(r, \theta)} \frac{\partial}{\partial t}, \quad X_{5}=e^{-\frac{1}{2} B(r, \theta)}\left(r e^{B(r, \theta)-A(r, \theta)} \frac{\partial}{\partial t}+t \frac{\partial}{\partial r}\right)
\end{aligned}
$$




$$
\begin{aligned}
& X_{6}=e^{-\frac{1}{2} B(r, \theta)}\left(\phi \frac{\partial}{\partial r}-r \frac{\partial}{\partial \phi}\right) \\
& X_{7}=e^{-\frac{1}{2} B(r, \theta)}\left(r \frac{\partial}{\partial \theta}-\theta \frac{\partial}{\partial r}\right), X_{8}=e^{-\frac{1}{2} B(r, \theta)}\left(\theta \frac{\partial}{\partial \phi}-\phi \frac{\partial}{\partial \theta}\right), \quad X_{9}=e^{-\frac{1}{2} B(r, \theta)} \frac{\partial}{\partial \theta} \\
& X_{10}=e^{\frac{1}{2} B(r, \theta)-A(r, \theta)} \frac{\partial}{\partial \phi} .
\end{aligned}
$$

4. Conclusion. In this paper we examine the teleparallel Killing vector fields of special axially symmetric static spacetimes in teleparallel theory of gravitation by using direct integration and algebraic techniques. We discussed the three different conditions under different constraints in which two of them give contradiction, while one gives the solution of the system comprising the killing equations in the form of killing vector fields. We observed that the dimension of the killing symmetry is 10 in the third condition.

\section{REFERENCES}

[1]. Petrov, A. Z. (1969). Physics Einstein spaces. Pergamon, Oxford University Press.

[2]. Ali, A., Hussain, T. and Khan, J. (2012). Isometries in Bianchi types $V I I_{0}$ and VII 0 space-times.Advanced Studies in Theoretical Physics. 6, 607-613.

[3]. Ali, A., Kamran and Minullah, Z. (2012). Classification of spherically symmetric non static spacetimes according to their Killing vector fields. Applied Mathematical Sciences. 6, 2681-2686.

[4]. Ali, A., Khan, J. and Hussain, T. (2012). Proper homothetic symmetry in Bianchi type IV spacetimes. Advanced Studies in Theoretical Physics. 6, 187-192.

[5]. Ali, A., Minullah Z. and Kamran. (2012). Proper homothetic vector field in Bianchi type V spacetimes,Advanced Studies in Theoretical Physics. 6, 193-200.

[6]. Shabbir, G., Ramzan, M. and Ali, A. (2009). Classification of non static spherically symmetric space-times according to their proper conformal vector fields. University Polithnica of Bucharest scientific bulletin series A-Applied Mathematics and Physics. 71(1), 3-8.

[7]. Shabbir, G. and Ali, A. (2009). A note on proper conformal symmetry in Bianchi types VIII and IX space-times. Advanced Studies in Theoretical Physics. 3, 93-97.

[8]. Shabbir, G. and Ali, A. (2009). Classification of spatially homogeneous rotating space-times according to their conformal vector fields. Applied Mathematical Sciences. 3(18), 869-876.

[9]. Shabbir, G. and Khan, S. (2010). A note on self similar vector fields in plane symmetric static spacetimes. TWMS Journal of Pure and Applied Mathathematics. 1, 252-256.

[10]. Shabbir, G. and Khan, S. (2012). A note on self-similar vector fields in static spherically symmetric spce-times. University Polithnica of Bucharest scientific bulletin series A-Applied Mathematics and Physics. 74(4), 177-182.

[11]. Shabbir, G. and Khan, S. (2013). A note on self-similar vector fields in cylindrically symmetric static space-times. TWMS Journal of Pure and Applied Mathematics. 04, 38-43.

[12]. Aldrovandi, R. and Pereira, J. G. (1995). An introduction to geometrical physics, World Scientific.

[13]. Sharif, M. and Amir, M. J. (2008). Teleparallel Killing vectors of the Einstein universe. Modern Physics Letters A. 23(13), 963-969.

[14]. Shabbir, G., Khan, S. and Ali, A. (2011). A note on classification of spatially homogeneous rotating space-times according to their teleparallel Killing vector fields in teleparallel theory of gravitation. Communications in Theoretical Physics, 55(2), 268-272.

[15]. Shabbir, G., Ali, A. and Khan, S. (2011). A note on teleparallel Killing vector fields in Bianchi type VIII and IX space-times in teleparallel theory of gravitation. Chinese Physics B. 20, 070401-070406.

[16]. Shabbir, G. and Khan, S. (2010). A note on Killing vector fields of Bianchi type II space- times in teleparallel theory of gravitation. Modern Physics Letters A. 25, 1733-1740.

[17]. Shabbir, G. and Khan, S. (2010). Classification of cylindrically symmetric static space-times according to their Killing vector fields in teleparallel theory of gravitation. Modern Physics Letters A. 25, 525-533.

[18]. Shabbir, G., Khan, S. and Amir, M. J. (2011). A note on classification of cylindrically symmetric non 
static space-times according to their teleparallel Killing vector fields in the teleparallel theory of gravitation. Brazilian Journal of Physics. 41, 184-194.

[19]. Shabbir, G. and Khan, S. (2010). Classification of Bianchi type I space-times according to their proper teleparallel homothetic vector fields in the teleparallel theory of gravitation. Modern Physics Letters A. $25,2145-2153$.

[20]. Shabbir, G. and Khan, S. (2010). Classification of teleparallel homothetic vector fields in cylindrically symmetric static space-times in teleparallel theory of gravitation. Communications in Theoretical Physics. 54, 675-678.

[21]. Shabbir, G. and Khan, S. (2012). A note on proper teleparallel homothetic vector fields in non static plane symmetric Lorentzian manifold. Romanian Journal of Physics. 57, 571-581.

[22]. Khan, S., Hussain, T. and Khan, G. A. (2013). A note on proper teleparallel homothetic motions of well-known spacetime using non diagonal tetrad. Life Science Journal. 10 (11s), 87-90.

[23]. Aldrovendi, R. and Pereira, J. G. (2001). An introduction to gravitation theory. Preprint.

[24]. Stephani, H., Kramer, D., Maccallum, M., Hoenselaers C. and Herlt, E. (2003). Exact Solutions of Einstein's Field Equations (Second Edition), Cambridge University Press. 